\title{
Identification of Potential Prognostic Biomarkers for Breast Cancer Based on lncRNA-TF-Associated ceRNA Network and Functional Module
}

\author{
Xinrong Li $\mathbb{D}$, Junquan Zhu $\mathbb{D}^{D}$, and Jian Qiu \\ Department of Integrative Medicine \& Medical Oncology, Shengzhou People's Hospital (The First Affiliated Hospital of Zhejiang \\ University Shengzhou Branch), 312400, Shengzhou, Zhejiang, China
}

Correspondence should be addressed to Jian Qiu; qj3887113@163.com

Received 30 April 2020; Revised 23 June 2020; Accepted 29 June 2020; Published 29 July 2020

Academic Editor: David A. McClellan

Copyright (c) 2020 Xinrong Li et al. This is an open access article distributed under the Creative Commons Attribution License, which permits unrestricted use, distribution, and reproduction in any medium, provided the original work is properly cited.

\begin{abstract}
Breast cancer leads to most of cancer deaths among women worldwide. Systematically analyzing the competing endogenous RNA (ceRNA) network and their functional modules may provide valuable insight into the pathogenesis of breast cancer. In this study, we constructed a lncRNA-TF-associated ceRNA network via combining all the significant lncRNA-TF ceRNA pairs and TF-TF PPI pairs. We computed important topological features of the network, such as degree and average path length. Hub nodes in the lncRNA-TF-associated ceRNA network were extracted to detect differential expression in different subtypes and tumor stages of breast cancer. MCODE was used for identifying the closely connected modules from the ceRNA network. Survival analysis was further used for evaluating whether the modules had prognosis effects on breast cancer. TF motif searching analysis was performed for investigating the binding potentials between lncRNAs and TFs. As a result, a lncRNA-TF-associated ceRNA network in breast cancer was constructed, which had a scale-free property. Hub nodes such as MDM4, ZNF410, AC0842-19, and CTB-89H12 were differentially expressed between cancer and normal sample in different subtypes and tumor stages. Two closely connected modules were identified to significantly classify patients into a low-risk group and high-risk group with different clinical outcomes. TF motif searching analysis suggested that TFs, such as NFAT5, might bind to the promoter and enhancer regions of hub lncRNAs and function in breast cancer biology. The results demonstrated that the synergistic, competitive lncRNA-TF ceRNA network and their functional modules played important roles in the biological processes and molecular functions of breast cancer.
\end{abstract}

\section{Introduction}

Breast cancer is one of the most common female cancers worldwide, which is also the second leading cause of female cancer death [1]. Adjuvant therapy has been an effective way to improve patient survival and promote the quality of life [2]. However, tumor metastasis and drug resistance are still a concern during breast cancer therapy. Thus, there is an urgent need to identify key biomarkers and uncover potential molecular mechanisms for breast cancer diagnosis and therapy. Many studies have identified some important genes that participated in the occurrence, development, and metastasis of breast cancer. For example, two well-known cancer genes, BRCA1 and BRCA2, were the major genes associated with the genetic etiology of breast cancer. Women with BRCA1/BRCA2 mutations had very high risk to develop breast cancer [3]. Mutations or variants of other genes such as TP53, ATM, BARD1, CHECK2, FGFR2, GSTM1, and MAP3K1 have also been reported to increase the risk of breast cancer [4].

Long noncoding RNAs (lncRNAs) are a type of RNA transcript of more than 200 nucleotides, which have been considered effective disease biomarkers in cancers [5]. Abnormal expression of several lncRNAs has been shown to be involved in breast cancer. For example, lncRNA HOTAIR was overexpressed and acted as a powerful predictor of metastasis in breast cancer [6]. The depletion of lncRNA MALAT1 decreased the tumorigenesis and 
metastasis of breast cancer [7]. IncRNA AGAP2-AS1 could promote breast cancer cell growth by upregulating the expression of $M y D 88$ and activating the NF- $\kappa \mathrm{B}$ signaling pathway [8]. In addition to these important functions in breast cancer, many recent studies have reported that lncRNAs might interact with mRNAs, competitively bind to their common microRNAs (miRNAs), and then function as competing endogenous RNAs (ceRNAs) [9]. The ceRNA-related network could link the functions of lncRNAs, miRNAs, and mRNAs. Dysfunction of these molecules in the network was highly related to the occurrence and development of human diseases, including breast cancer [10].

Although a single gene can function in the study of pathogenesis, detection of individual gene expression can still not promote the overall understanding of human diseases [11]. Recently, the application of biological networks for identifying biomarkers and understanding cancer biology has become increasingly urgent [12]. Networks specific to disease context could help in improving the understanding of the underlying biology from a global perspective [13]. Transcription factors (TFs) are a kind of genes that could function in the regulation of gene expression via binding to their DNA regulatory elements, such as promoters or enhancers [14]. The miRNAs, TFs, and the mRNAs or lncRNAs regulated by them could be integrated for constructing global regulatory networks. More intriguingly, network module centrality analysis provided more information to understand biological problems [15]. However, some regulatory patterns such as lncRNA-TF interactions in breast cancer remained unknown. More important molecular mechanisms underlying breast cancer still need more comprehensive molecular and biological studies.

In the present study, we are working to construct a lncRNA-TF-associated ceRNA network for revealing their potential interaction in breast cancer using bioinformatics tools. This network contained TFs, lncRNAs, and their interactions based on ceRNAs and protein-protein interactions (PPIs). First, we performed a comprehensive analysis of the network and computed important topological features, such as degree and average path length. Hub nodes with the highest degrees in the IncRNA-TF-associated ceRNA network were selected to detect differential expression in different subtypes/tumor stages of breast cancer. Then, closely connected modules were identified from the IncRNA-TF-associated ceRNA network. Survival analysis was performed to evaluate whether the modules had prognosis effects on breast cancer. Furthermore, in order to investigate the binding potential between TFs and lncRNAs, we performed TF motif searching to indicate the promoter and enhancer regions of lncRNAs. In conclusion, our study could help explain the biological processes and molecular mechanism of breast cancer from a global network perspective.

\section{Materials and Methods}

2.1. Breast Cancer-Related Datasets. We downloaded breast cancer-related gene expression profile from TCGA (https:// xenabrowser.net/datapages/) and converted transcript-level
RNA-seq data into lncRNA/protein-coding gene-level RNA-seq data using GENCODE (https://www .gencodegenes.org/human/) [16]. TFs that we obtained before were further mapped to the protein-coding genelevel RNA-seq data. Data preprocessing and log transformation were performed to these RNA-seq data. Finally, breast cancer-related lncRNA/TF RNA-seq expression profiles were obtained. These data involved 1,215 samples with clinical information. All the raw expression data are supported in Supplementary Tables S1 and S2.

2.2. Construct a lncRNA-TF-Associated ceRNA Network. Based on ceRNA theory, we comprehensively analyze lncRNA and TF RNA-seq expression profiles of breast cancer and constructed a lncRNA-TF-associated ceRNA network. First, we downloaded all the miRNA-mRNA interactions that were curated from StarBase (http://starbase.sysu.edu .cn/), which contained 386 miRNAs and 13,861 mRNAs (supported in Supplementary Table S3). The miRNA-TF interactions were further extracted by mapping TFs to the mRNAs obtained previously. In addition, we used miRanda tools for identifying significant miRNA-lncRNA interactions by inputting lncRNA and miRNA sequences (default parameters) [16]. Second, we counted the number of the shared miRNAs for each lncRNA-TF pair based on the miRNA-TF interactions and miRNA-lncRNA interactions and indicate the shared miRNAs with statistical significance for all the lncRNA-TF pairs using hypergeometric test. The lncRNA-TF pairs with the threshold of hypergeometric test $p$ value $<0.05$ were considered statistically significant (Supplementary Table S4).

Third, using breast cancer-related lncRNA and TF-level RNA-seq expression profiles, Pearson correlation coefficients (PCC) were further calculated for those lncRNA-TF pairs with a hypergeometric test $p$ value $<0.05$. And the lncRNA-TF pairs with PCC $>0.6$ were finally considered significant lncRNA-TF pairs (Supplementary Table S5).

In addition, TF-related PPI interactions were extracted from the HPRD database. Then, a breast cancer-related lncRNA-TF ceRNA network was formed by combining all the significant lncRNA-TF pairs and TF-TF PPI pairs (Supplementary Table S6).

2.3. Identify Closely Connected Network Modules. We used the Molecular Complex Detection (MCODE) plug-in in Cytoscape to identify closely connected modules from the lncRNA-TF-associated ceRNA network. The MCODE algorithm is based on graph-theoretical analysis, which clusters a given network by topology for finding densely connected regions [17]. The criteria that we used for identifying functional modules were as follows: MCODE scores $>5$, degree cutoff $=2$, node score cutoff $=0.2$, $\max$ depth $=100$, and $k-$ score $=c 2$.

2.4. Survival Analysis. Our gene expression profile contained 1,215 breast cancer patients with clinical information. Subtype classification is defined from TCGA clinical matrix. Based on these data, the univariate Cox regression was used to identify breast cancer-related prognostic signatures. We 
accumulated the regression coefficient and the expression values of each gene and computed the risk score of each patient as follows:

$$
\text { Risk score }=\sum_{i=1}^{n} r_{i} \operatorname{Exp}(i) \text {, }
$$

where $n$ is the number of genes in a gene set, $r_{i}$ is the regression coefficient of gene $i$, and $\operatorname{Exp}(i)$ is the expression value of gene $i$ for a corresponding patient.

We classified breast cancer patients into two groups by using the mean risk score as a cutoff. That is, patients with the risk score greater than the mean value were classified into a high-risk group. Patients with the risk score less than the mean value were classified into a low-risk group. These high-risk group and low-risk group patients were then used to perform Kaplan-Meier survival analysis. Log-rank test with a $p$ value $<0.05$ was used to generate statistical significance. The raw TCGA clinical matrix is supported in Supplementary Table S7.

2.5. TF Motif Searching Analysis. For investigating the binding potential between TFs and lncRNAs, we performed TF motif searching analysis to the promoter and enhancer regions of lncRNAs. Promoters were defined as $+/-2 \mathrm{~kb}$ from transcription start site (TSS). Enhancers were downloaded from FANTOM5 $[18,19]$. FIMO was used to scan promoter and enhancer regions with a $p$ value $<1 e-4[20]$.

\section{Results}

3.1. Construction of a IncRNA-TF-Associated ceRNA Network. IncRNAs that contained miRNA-response elements could competitively bind miRNAs with mRNAs and then function as ceRNAs to participate in multiple biological processes of complex diseases. In this study, a IncRNA-TF-associated ceRNA network in breast cancer was constructed by combining all significant lncRNA-TF ceRNA pairs and TF-TF PPI pairs (Figure 1, details in methods). This network consisted of 164 lncRNA nodes, 91 TF nodes, and 644 edges (Figure 2(a)). To evaluate the importance of network nodes, we performed topological analyses for the lncRNA-TF-associated ceRNA network (Supplementary Table S8). First, we computed the degree of network nodes and found that all the nodes followed a power law distribution, which indicated that the network had the scale-free property (Figure 2(b), $R^{2}=$ 0.94). Next, we calculated the average path length of the lncRNA-TF-associated ceRNA network. Simultaneously, we also chose 1,000 degree-conserved random networks to calculate their average path length and counted the number of average path length in a random network shorter than that in the real network. $p$ values were calculated by the number divided by 1,000 . The result showed that the average path length of the real network was significantly shorter than that of random networks (Figure 2(c), $p<0.01)$. These results suggested that hub genes of the lncRNA-TF-associated ceRNA network played important roles in the local region of the network.
3.2. Detection of Breast Cancer-Related Hub Genes. Numerous studies found that genes connected by a large number of other genes (also known as high degree) in biological network tended to play vital roles in pathological processes. These genes with high degree in network were defined as hub genes. Here, we detected breast cancerrelated hub genes from the IncRNA-TF ceRNA network. We defined the genes with top $10 \%$ node degree as hub genes, including 14 TFs and 11 lncRNAs (Figure 3(a)). Results showed that these hubs not only had high degrees but also had high betweenness, closeness, and low shortest path length, indicating that these genes might maintain the basic biological processes in cancer pathology (Figure 3(a)). We further extracted the hub-hub subnetwork from the IncRNA-TF-associated ceRNA network. As a result, the hub-hub subnetwork was composed of all these hubs and their 103 edges, including the known cancer-related IncRNAs MALAT1 and XIST (Figure 3(b)). Then, we tested the prognosis effects of the 14 hub TFs. Results showed that hazard ratios of these TFs in breast cancer of TCGA BRCA cohorts were not significant (Figure 3(c)). However, in luminal A subtype, these hub TFs showed a strong prognosis effect (Figure 3(d)). These results inspired us to investigate the function of hub genes in subtypes of breast cancer.

The results mentioned above showed that our IncRNA-TF-associated ceRNA network had the scale-free property, representing a small subset of high-degree nodes (also called hubs) that were connected by the most of other nodes. Thus, we selected 2 TF hubs (MDM4 and ZNF410) and 2 lncRNA hubs (AC084219 and CTB89H12) with the highest degrees from the lncRNA-TFassociated ceRNA network and detected their expression in various subtypes of breast cancer. The results showed that they could significantly be distinguished between breast cancer samples and normal samples (Figures 4(a)$4(\mathrm{~d})$ ) in different subtypes. Actually, MDM4 has been emerging as an important breast cancer biomarker and oncoprotein [21]. MDM4 was found highly expressed not only in normal breast epithelial cells but also in most luminal breast cancer [22]. MDM4 has also been suggested to promote triple-negative breast cancer metastasis [23]. Cancer cells and stromal/immune cells, such as cancerassociated fibroblasts, were the important parts of tumor microenvironment. ZNF410, also known as $A P A-1$, was a $\mathrm{TF}$ that regulated the expression of matrix-remodeling genes during fibroblast senescence [24]. Du et al. have shown the tumor-suppressive role of lncRNA CTB-89H12 and the expression regulation ability of PTEN in prostate cancer [25]. The above studies suggested that hub nodes in global lncRNA-TF network might play important roles in biological processes and molecular functions of breast cancer. We further calculated the expression of the two lncRNAs (AC084219 and CTB-89H12) in different tumor stages and found that they were differentially expressed in the advanced stage of tumor (Figure 4(e)).

3.3. Identification of Closely Connected Network Modules. Biological networks are often too large to interpret the 


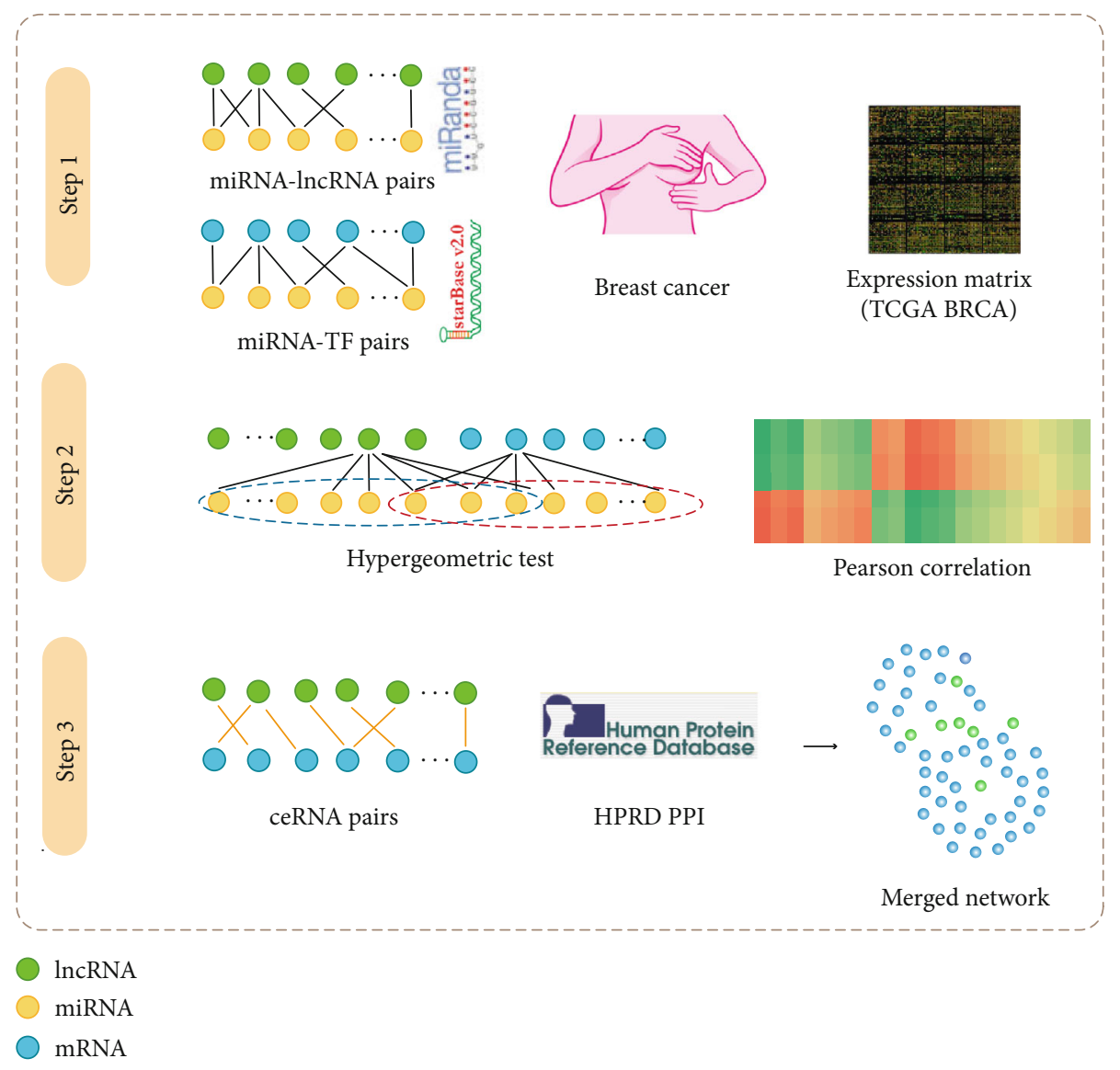

FiguRe 1

biological phenomena accurately. Functional modules of a network may be more useful for reflecting the relevant biological importance. Functional modules have been widely applied to explore the mechanism involved in various biological processes, such as miRNA regulation, disease occurrence, and drug action [26]. We used "MCODE" in the Cytoscape software to identify closely connected network modules from our lncRNA-TF-associated ceRNA network. As a result, two closely connected modules linked to breast cancer were identified.

Module 1 was composed of 43 nodes ( $26 \operatorname{lncRNAs}$ and 17 TFs) and 120 edges (Figure 5(a)). Some lncRNAs and TFs of module 1 have been reported to function in breast cancer. For example, MDM4 negatively regulated the major tumor suppressor gene $p 53$ and further modulated stress responses, which had been considered a biomarker that may drive metastasis and progression of breast cancer [27]. The altered expression of DMTF1 proteins was highly related to the pathophysiology of cancer. In response to oncogenic stresses, $D M T F 1$ bind to the promoter of $A R F$ and governed the $A R F-p 53$ tumor suppressor pathway activity [28]. LncRNA PURA was an evolutionarily conserved cellular protein participating in processes of DNA replication, transcription, and RNA transport, which functioned in human cancer [29]. To evaluate whether module 1 had prognosis effects on luminal A breast cancer, we calculated linear combination of expression values of lncRNAs/TFs in module 1 weighted by the regression coefficient of univariate Cox regression to perform survival analysis. As a result, we significantly classified luminal A breast cancer patients into low-risk group and high-risk group with different clinical outcomes (Figure 5(b)).

Module 2 was composed of 36 nodes ( $29 \operatorname{lncRNAs}$ and 7 TFs) and 45 edges (Figure 5(c)). We also found that several lncRNAs and TFs in module 2 were highly associated with breast cancer. MATR3 was a highly conserved nuclear matrix protein, which was widely expressed in various tissues and involved in breast cancer-related biological processes, such as transcription, translation, RNA processing, DNA replication, apoptosis, and chromatin remodeling [30]. Axitinib, a clinically approved drug, could effectively treat cancer patients with aberrant activity of nuclear $\beta$-catenin. The E3 ubiquitin ligase SHPRH was identified as the direct target of axitinib. Treatment with axitinib stabilized SHPRH and increased the ubiquitination and degradation of $\beta$-catenin [31]. Furthermore, we also calculated linear combination of expression values of lncRNAs/TFs in module 2 weighted by the regression coefficient of univariate Cox regression in order to evaluate whether module 2 had prognosis effects on luminal A and luminal B breast cancer. As shown in Figure 5(d), luminal A and luminal B breast cancer patients were significantly classified into low-risk group and highrisk group with different clinical outcomes, respectively. These results suggested that the integration of lncRNAs and 


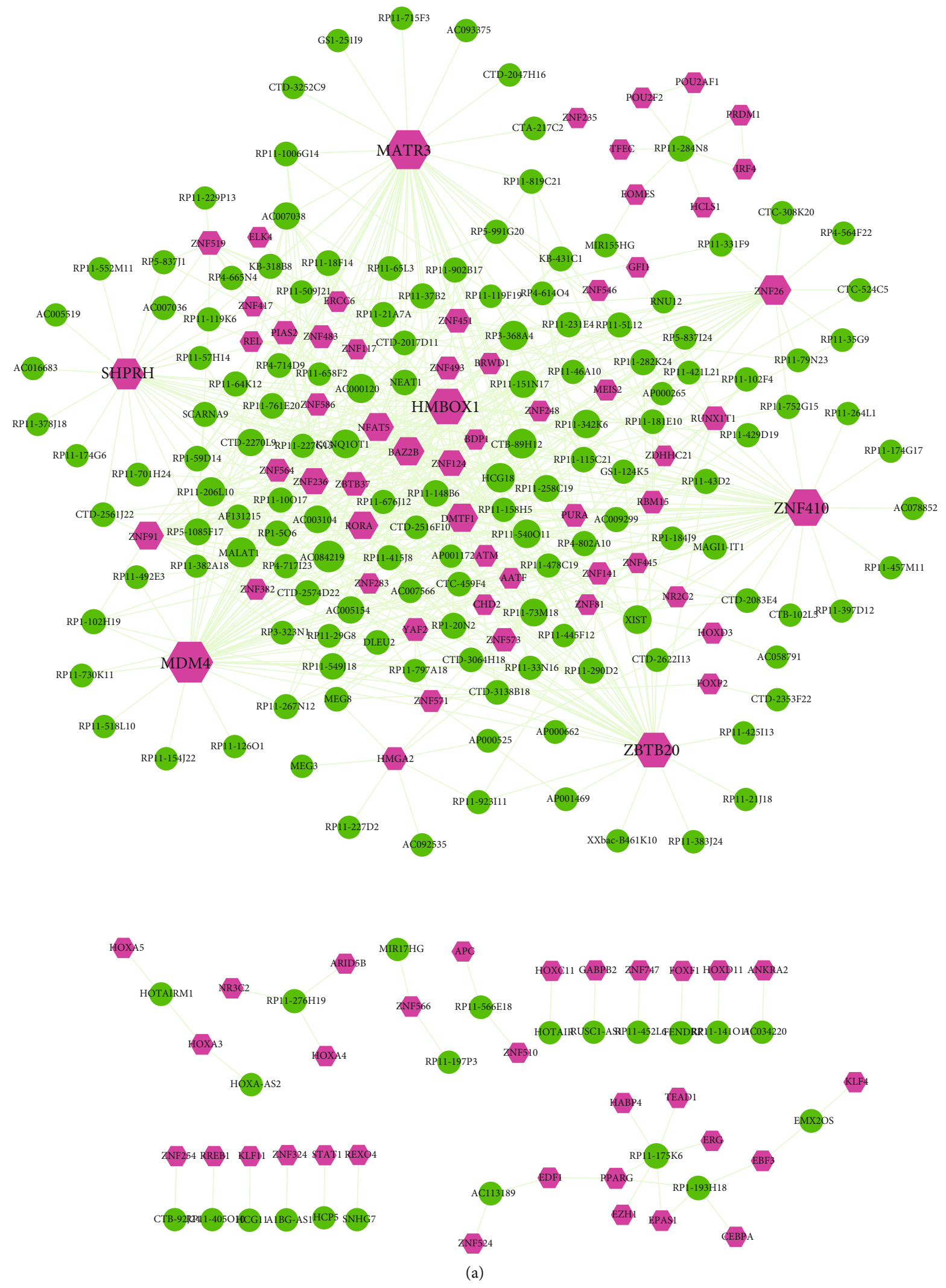

Figure 2: Continued. 


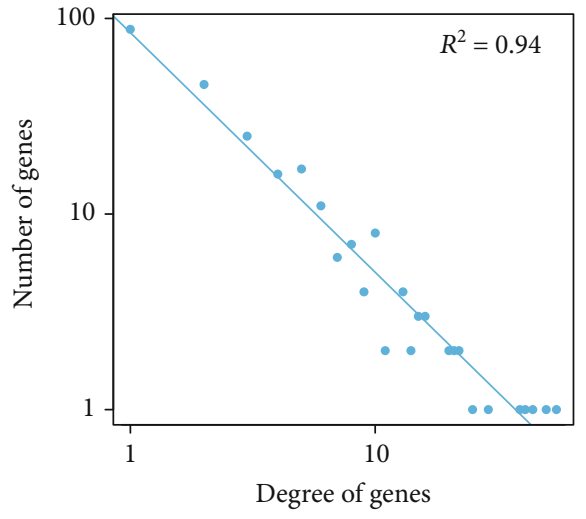

(b)

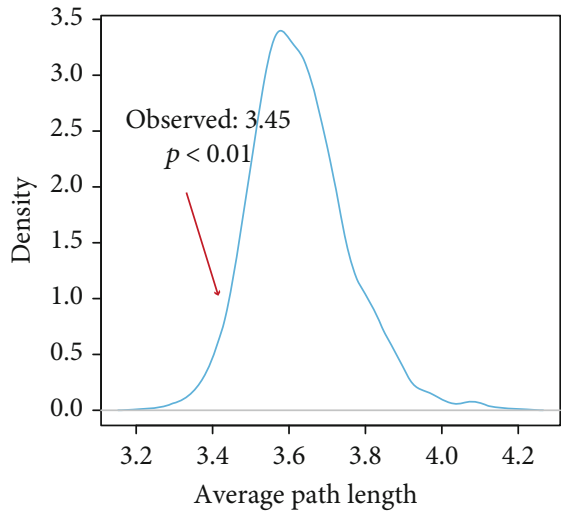

(c)

Figure 2

TFs in our functional modules had significant prognosis capability and could be used as prognostic signatures of breast cancer.

3.4. Identification of Core lncRNA-TF Crosstalks. TFs may control the activity of lncRNAs via binding to the DNA regulatory elements of lncRNAs. In this study, we conducted motif searching to the promoter and enhancer regions of lncRNAs for investigating the binding potential between TFs and lncRNAs. The results showed multiple TF binding sites in the promoters and enhancers of lncRNAs, respectively (Figures 6(a) and 6(b)). For example, NFAT5 has been implicated in cancer cell proliferation and invasion [32]. In this study, NFAT5 had ceRNA relationships with lncRNAs under the threshold of hypergeometric test $p$ value $<0.05$ and PCC $>0.6$, which were further validated to have multiple motifs binding in the promoters of lncRNAs (Figure 6(a)).

Because hub genes often play more important roles in the biological network, we focused on the motif searching results of top $20 \%$ hub $\operatorname{lncRNAs}$ in our breast cancerrelated lncRNA-TF ceRNA network. Those lncRNA-TF pairs with TFs binding in the promoters and enhancers of top $20 \%$ hub lncRNAs were extracted to form a new network (Figure 6(c)). That is, lncRNA nodes and TF nodes in this network had not only significant ceRNA relationships but also strong motif binding. The results implied that TFs might bind to the promoter and enhancer regions of important hub lncRNAs and form "feedback loops" to function in cancer biology. The results of KEGG pathway enrichment showed that TFs of the network were associated with basal functions, such as "Thyroid hormone signaling pathway," "Hepatitis B," "Transcriptional misregulation in cancer," "Pathways in cancer," and "Cell cycle" (Figure 6(d)). These pathways were all demonstrated to be closely associated with breast cancer [33-35]. For example, breast cancer patients during or after chemotherapy were found to have a remarkable clinical problem of hepatitis B virus [36]. In normal cells, thyroid hormones could regulate the normal physiological processes. However, once signaling pathways became dysregulated, thyroid hormones would induce cancer cell pro- liferation [37]. Insulin resistance that attenuated biological response to insulin circulation was reported to be associated with a series of pathological conditions and some endocrine tumors, including breast cancer [38]. All these results showed that TFs could crosstalk with lncRNAs via binding to the promoter and enhancer regions of lncRNAs, which were involved in breast cancer-related biological processes and molecular functions.

\section{Discussion}

Breast cancer is accountable for the plurality of cancer deaths among women worldwide. Metastatic breast cancer is even considered an incurable disease with poor prognosis [39]. There is an urgent need to investigate the molecular mechanism and find the significant risk factors for diagnosis and prognosis of breast cancer. The ceRNA regulation may represent a widespread layer of gene regulation which is important for pathogenesis such as breast cancer [40]. Systematically analyzing the lncRNArelated ceRNA network may provide valuable insight into the function of lncRNAs and the molecular mechanism of diseases. Thus, in this study, we constructed a global IncRNA-TF network for revealing their potential interaction in breast cancer using bioinformatics tools. This network was constructed by combining all significant lncRNA-TF ceRNA pairs and TF-TF PPI pairs. First, we made a comprehensive analysis of the network and computed important topological features, such as degree and average path length. We found that all the nodes followed power law distribution and average path length of the real network was substantially shorter than that of random networks. We selected hub nodes with the highest degrees in the global lncRNA-TF network and found that they could significantly distinguish between tumor samples of different subtypes/tumor stages and normal samples. The literature evidences further suggested the importance of hub nodes in the global lncRNA-TF network. Then, two closely connected modules containing some hub genes such as MDM4, DMTF1, RORA, and MATR3 were identified from the global lncRNA-TF network, which represented significant different 


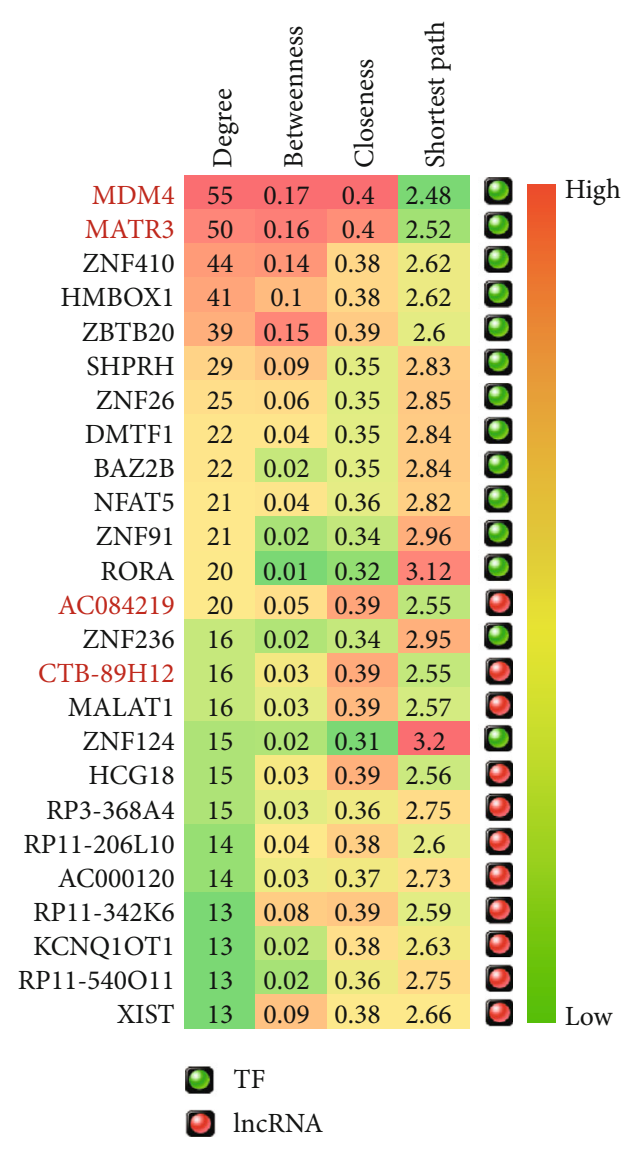

(a)

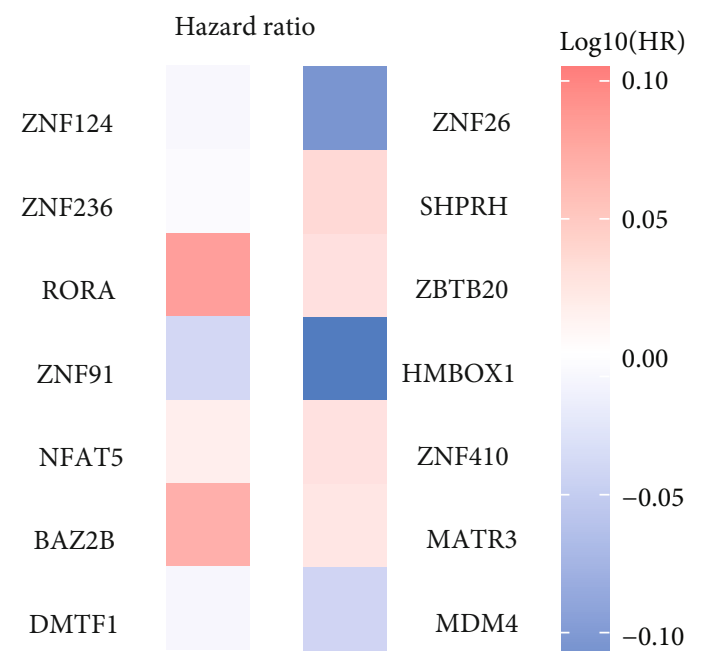

BRCA

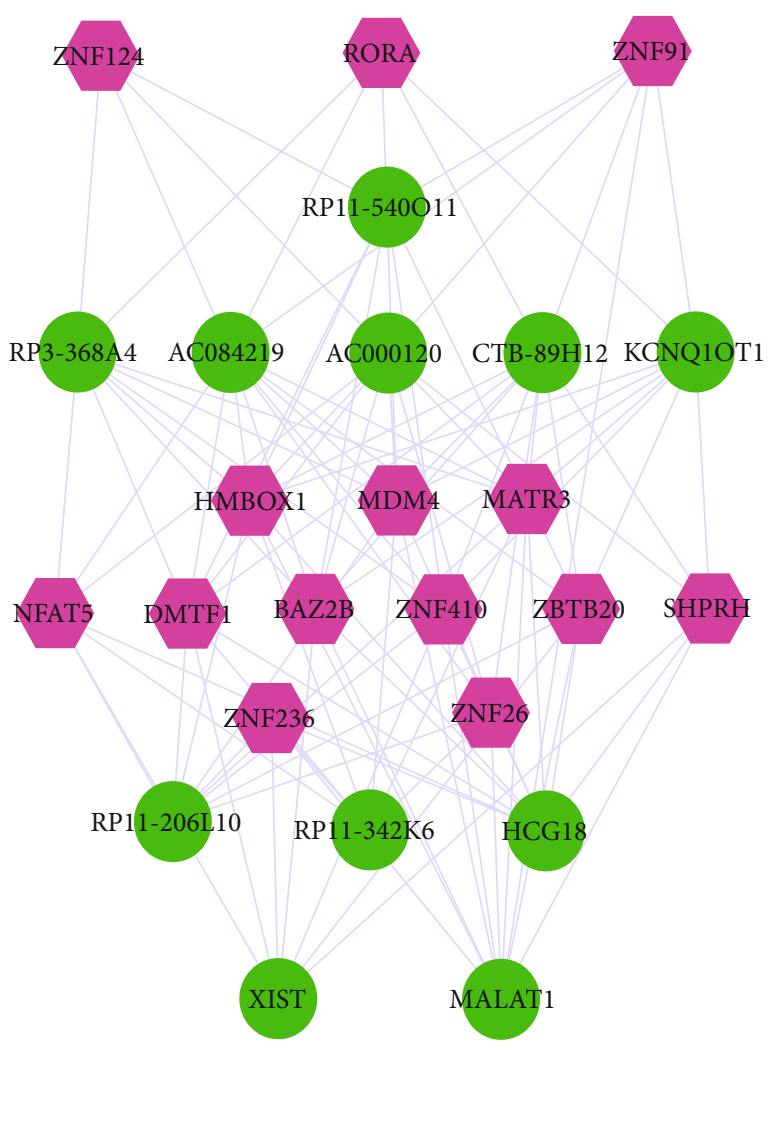

(b)

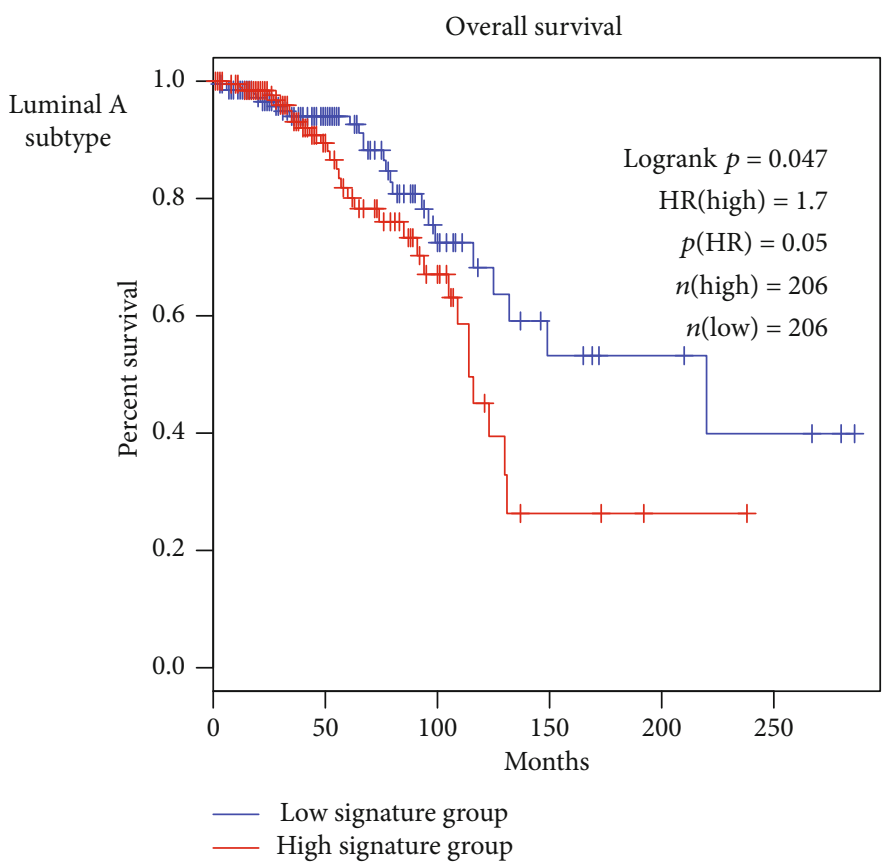

(d)

Figure 3 

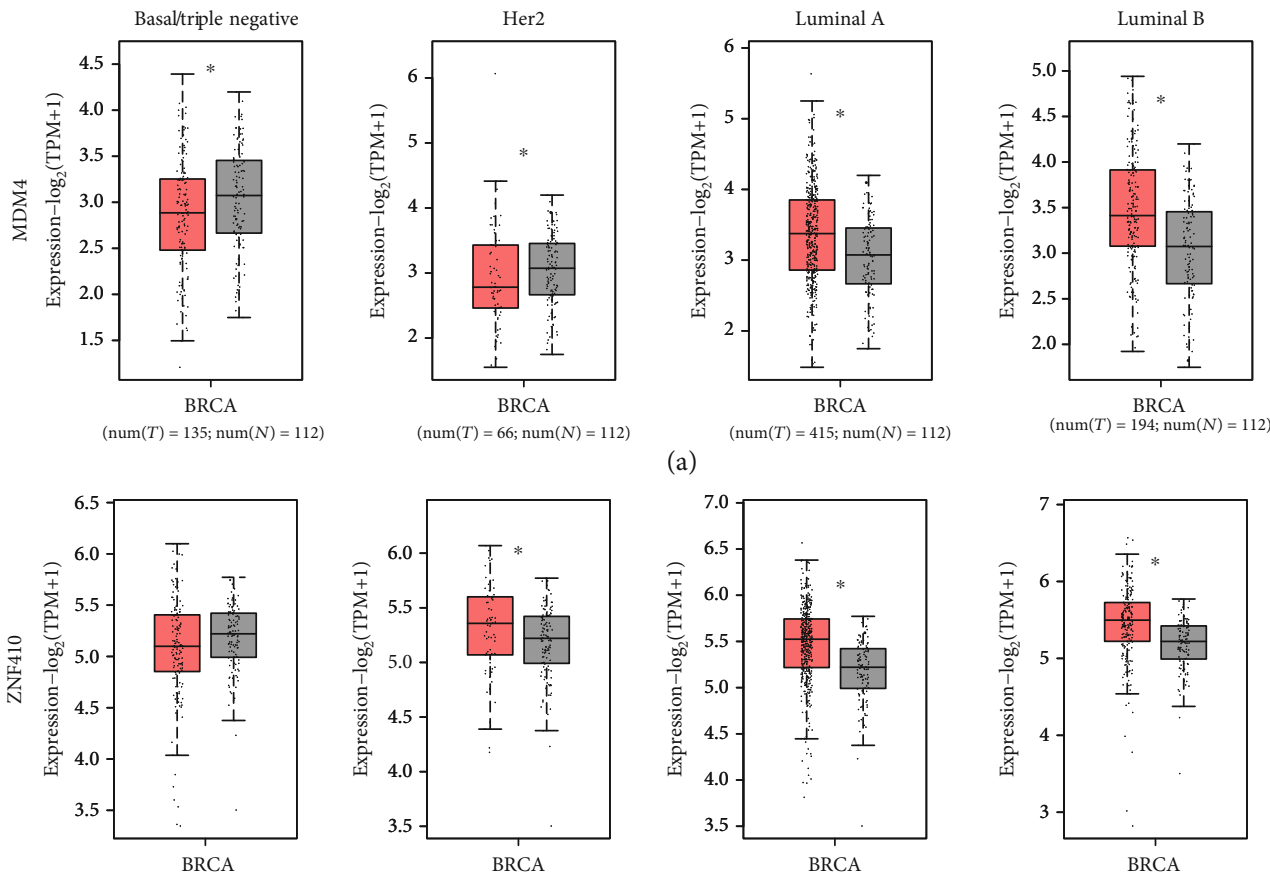

(a)
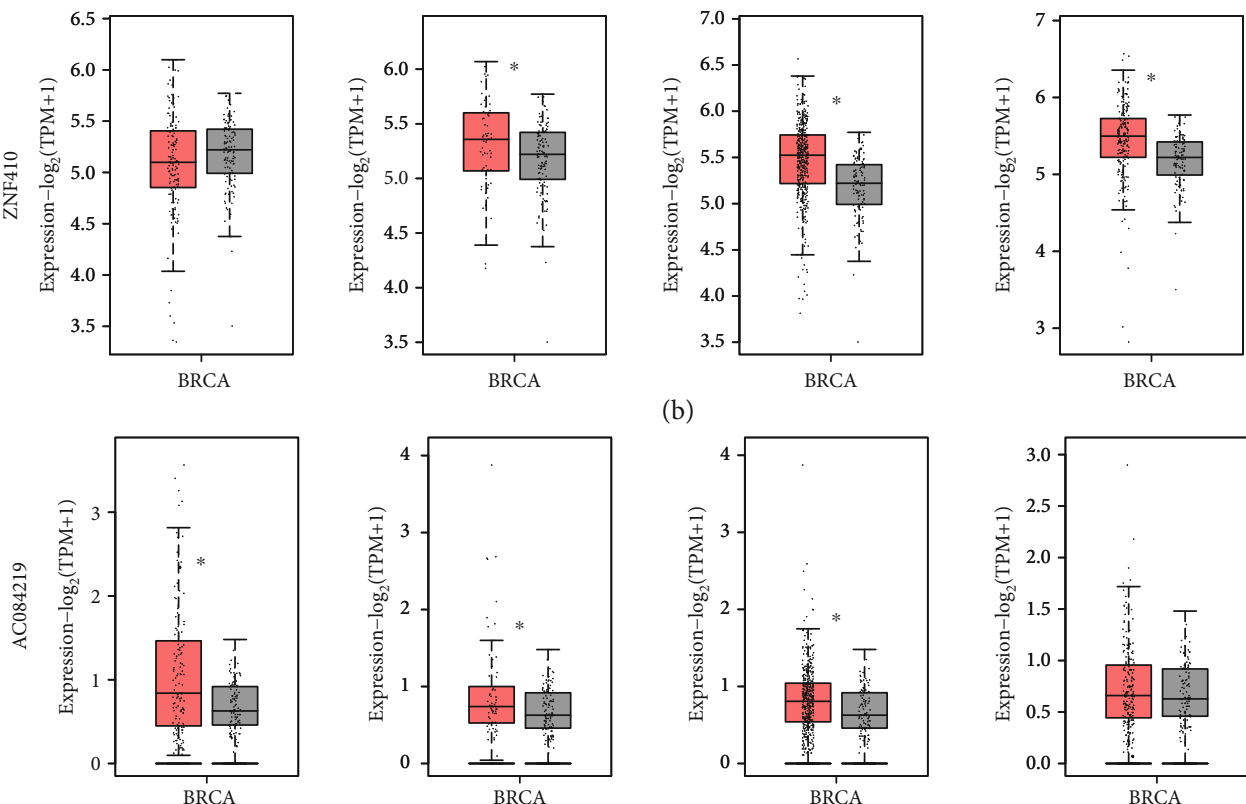

(b)
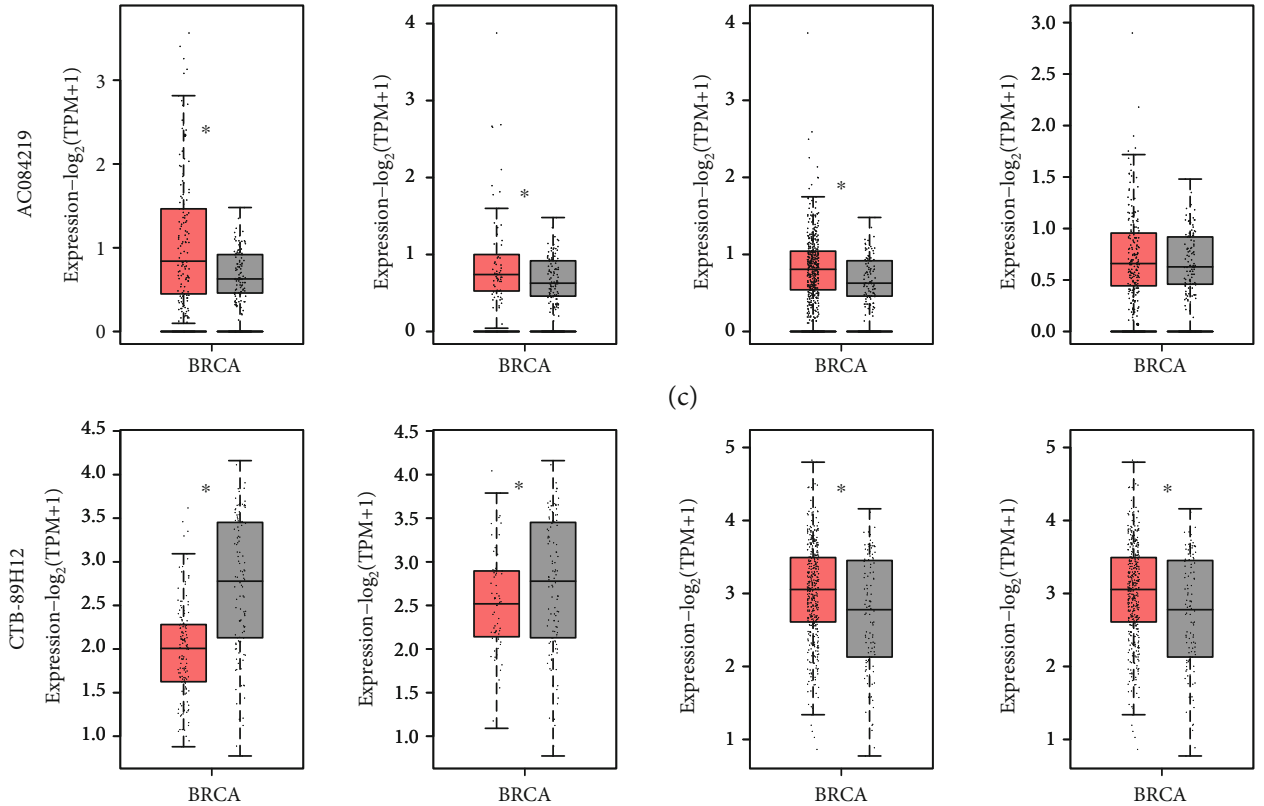

(c)
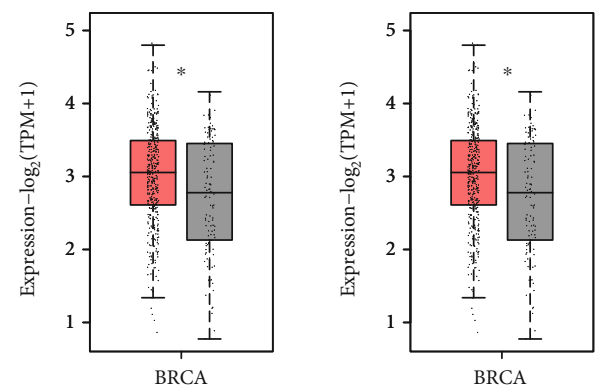

(d)
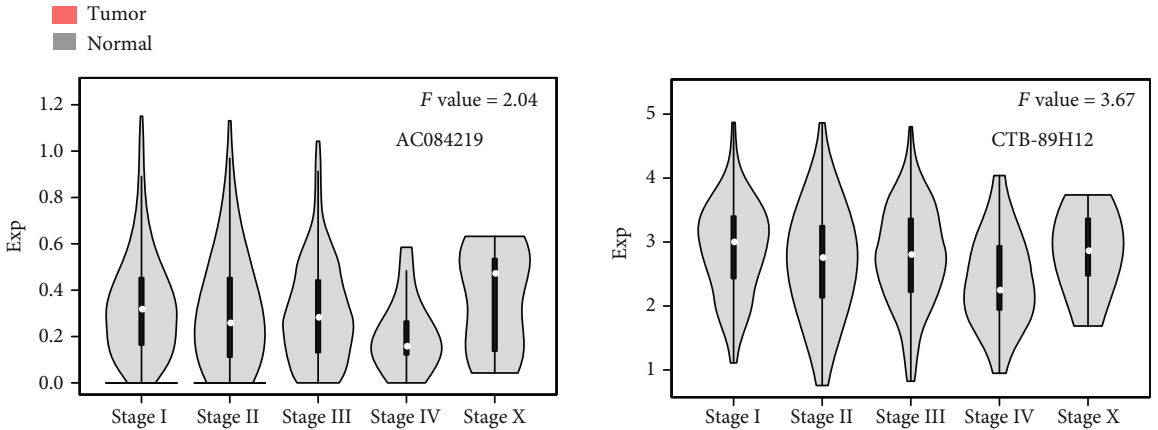

(e)

Figure 4 


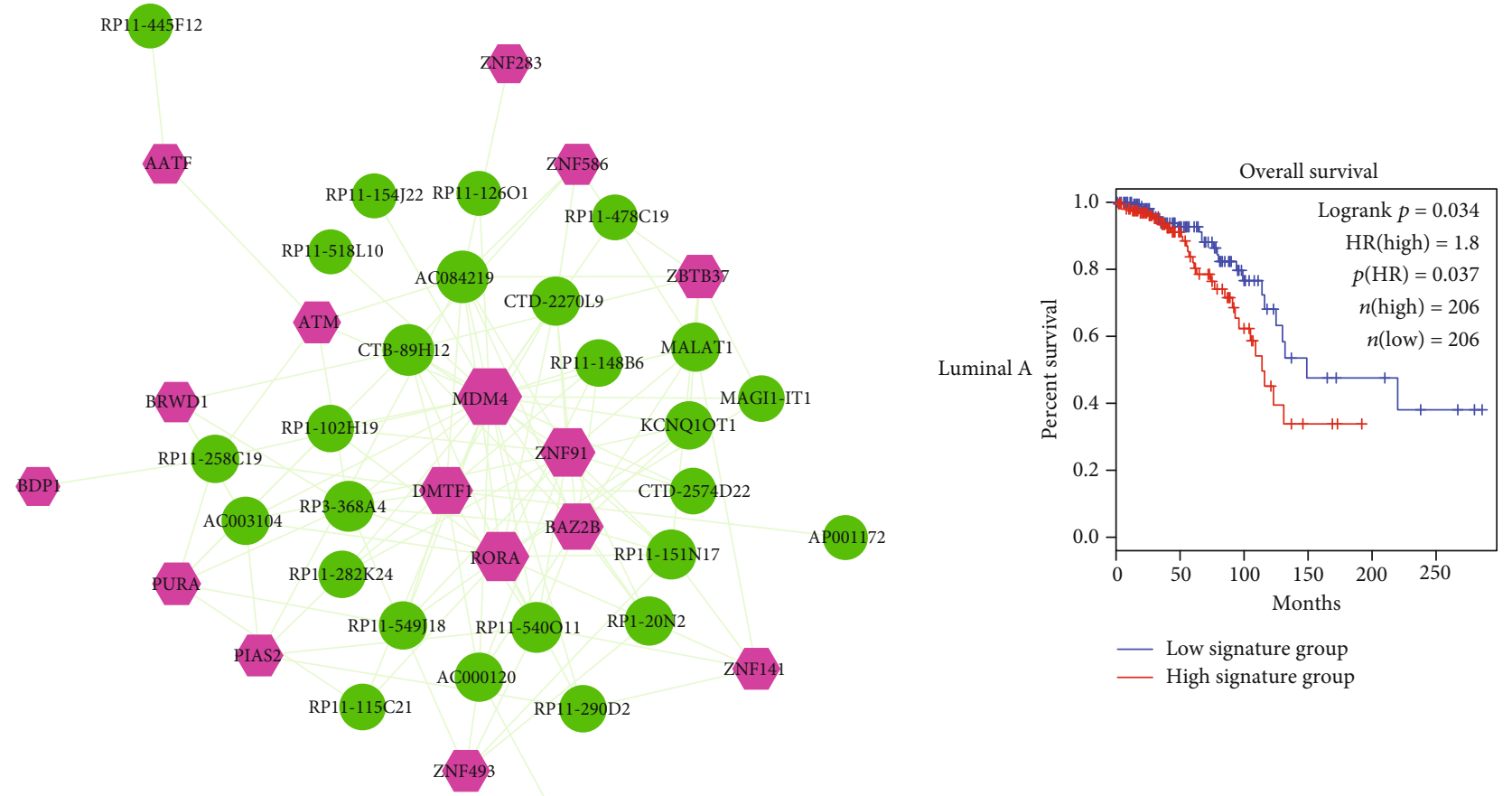

\section{ZNF117}

(a)

GS1-124K5

RP11-229P13<smiles>[CH]1CC[AsH2]C1</smiles>

AC007036

RP5-837J1

RP11-378)18

AC005519

AC016683
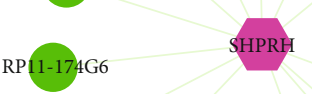

RP11-552M11

RP11-227G15
RP4-665N4

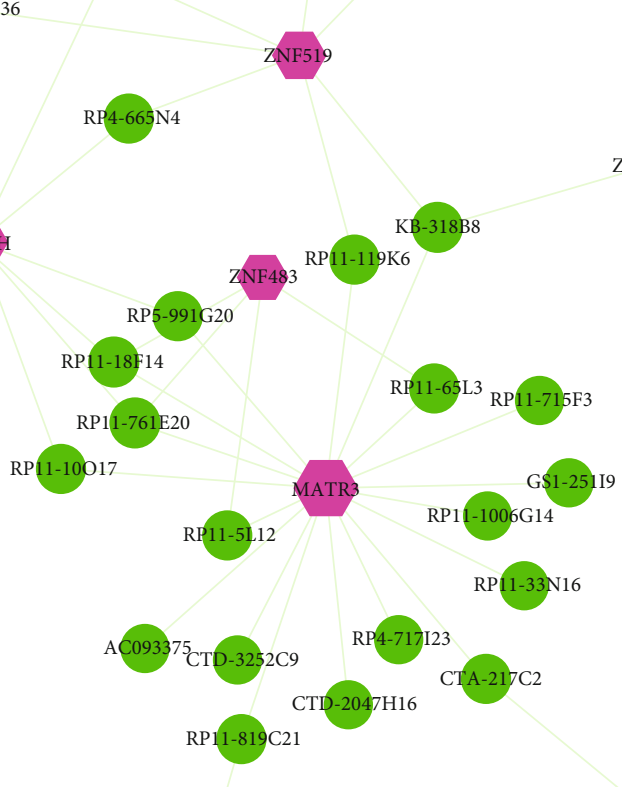

ZNF23

ZNF4) 7

(b)
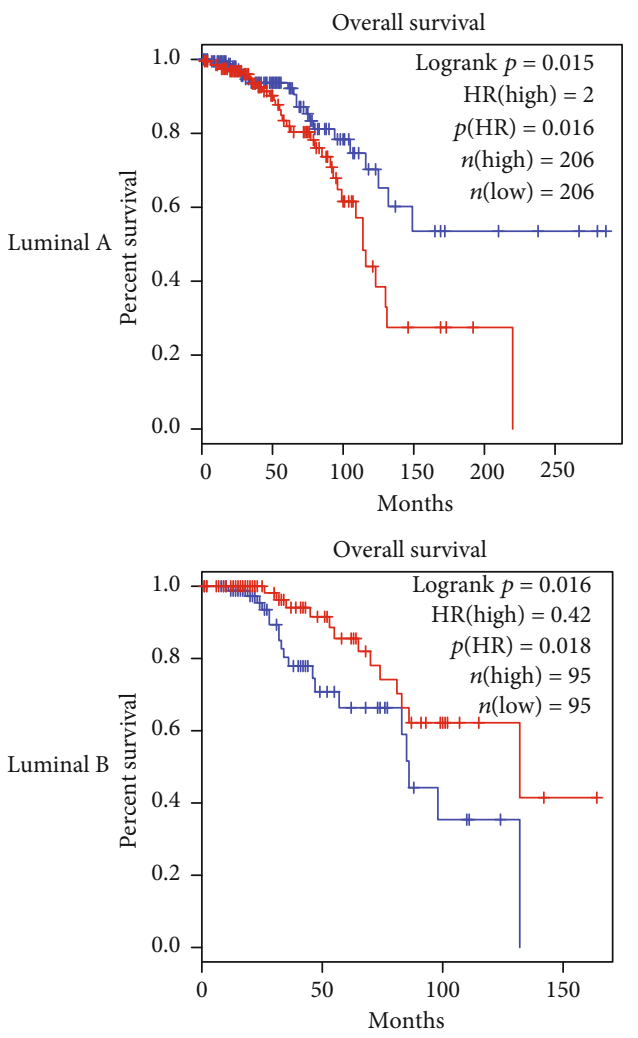

— Low signature group — High signature group

(d)

Figure 5 


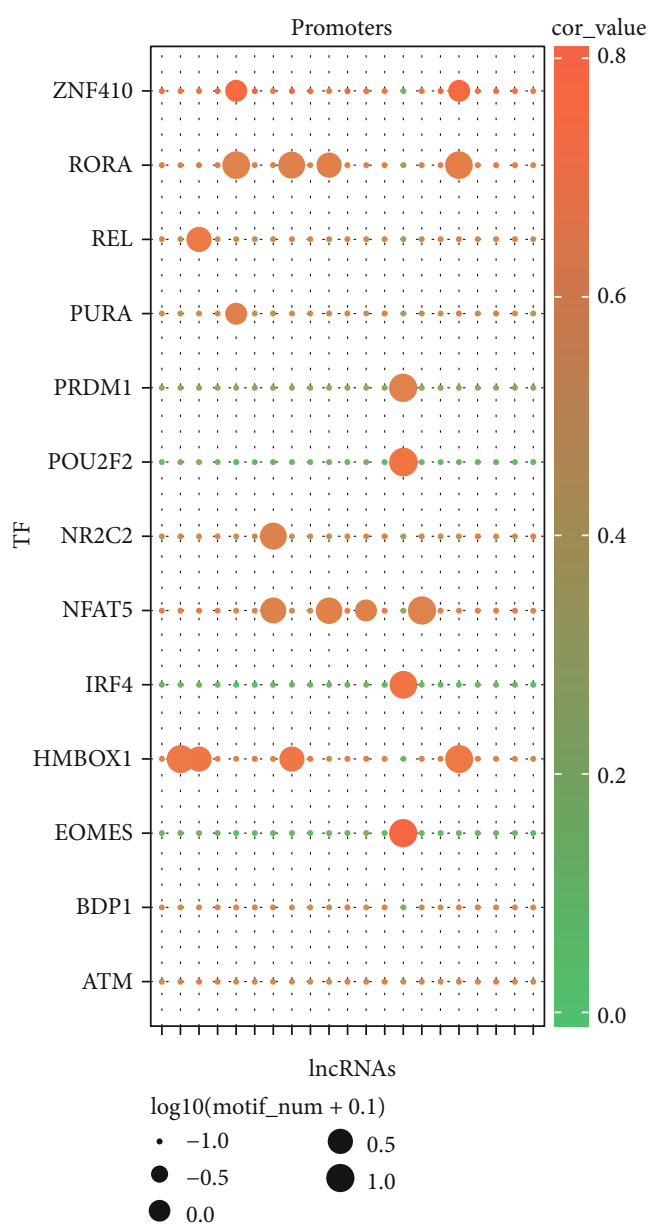

(a)

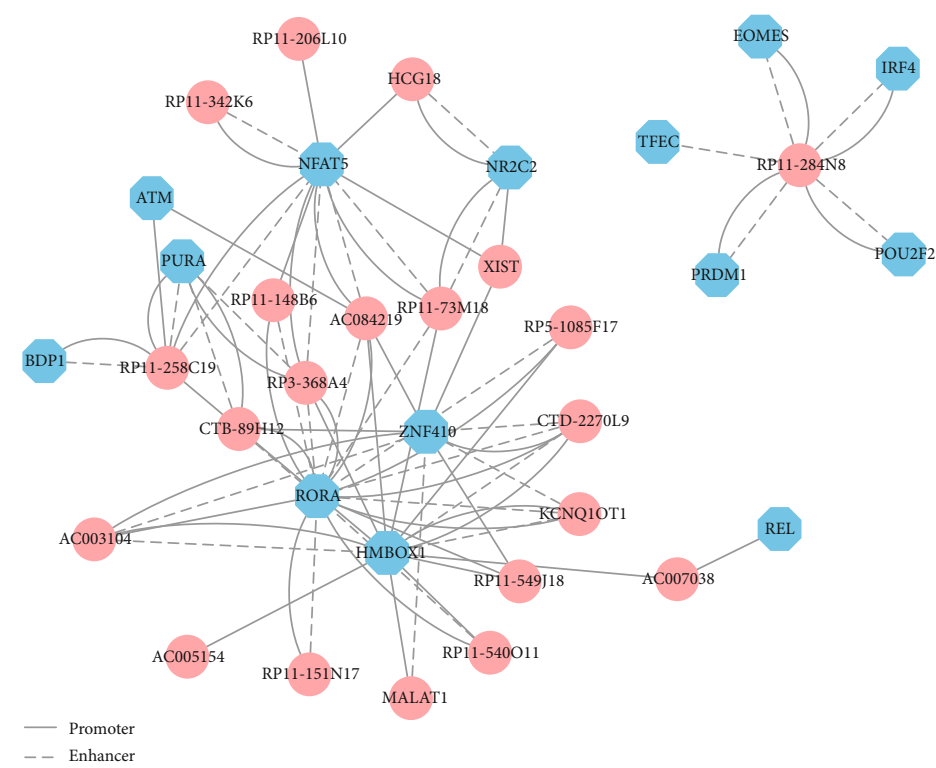

(c)

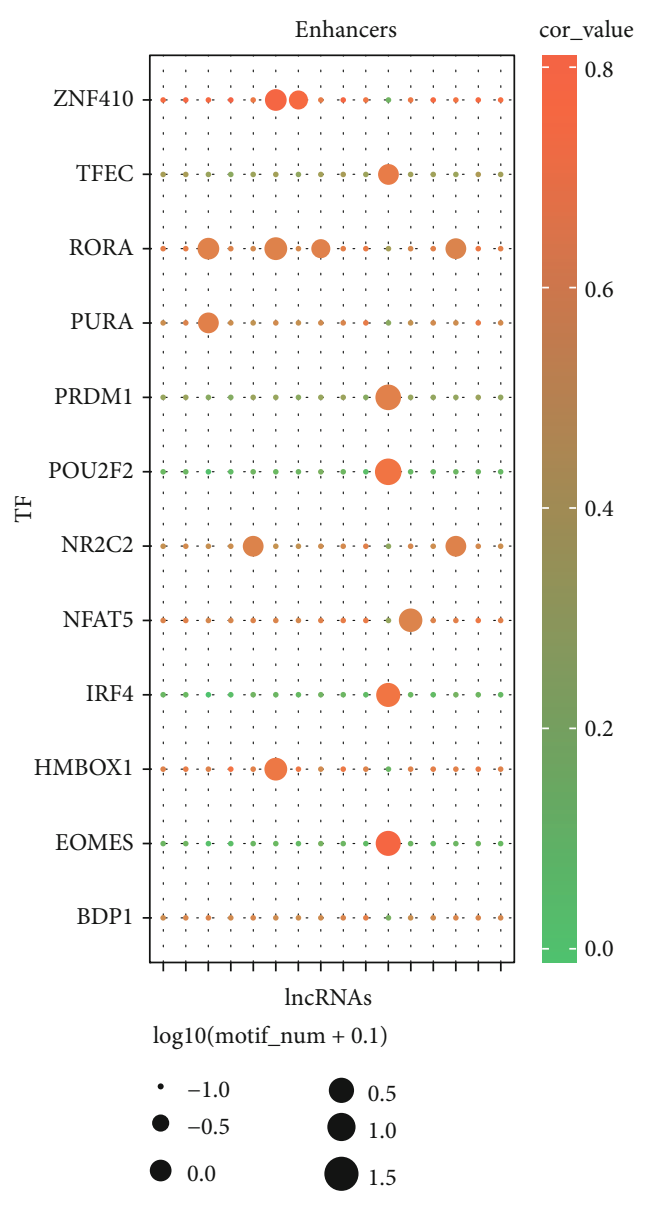

(b)

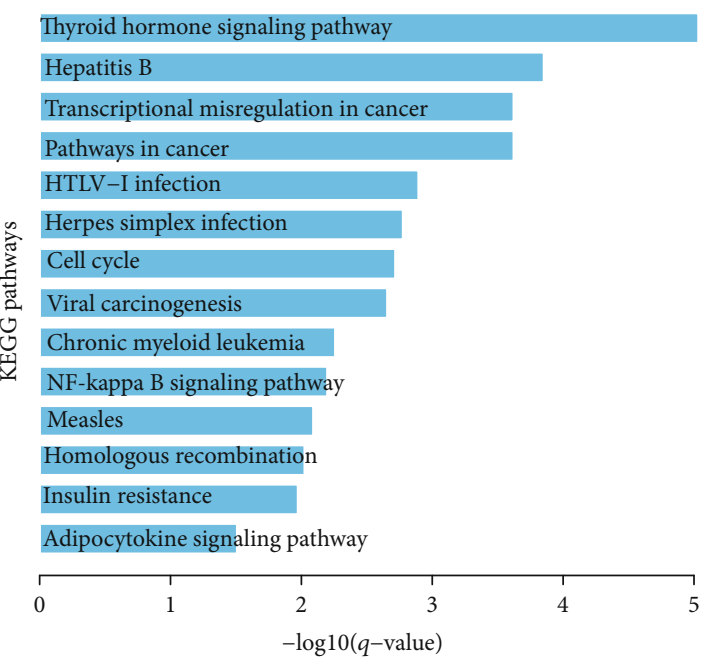

(d)

Figure 6

clinical outcomes between the breast cancer patients in the low-risk group and high-risk group classified by the survival analysis.
Here, as a point of innovation, we identified some subtype-specific prognosis factors in breast cancer. In Figure 3(c), results showed that TFs have the weak prognosis 
effects on panbreast cancer. However, combining these factors showed a strong prognosis effect in the luminal A subtype, which indicated that these crucial genes have an important clinical value in luminal A breast cancer. As previously mentioned, $M D M 4$, which is a negative regulator of $p 53$, not only played crucial roles in regulation of normal breast development but also contributed to the relapsing and metastasis of breast cancer. Intriguingly, MDM4 was significantly overexpressed in the luminal A subtype of breast cancer [41]. Thus, several anticancer therapeutic strategies such as SAR405838 [42], DS-3032b [43], and ALRN-6924 [44] were explored with the purpose of restoring the normal activity of p53. As for the DMTF1 in module 1, Tian et al. found that DMTF1 $\beta$, a major subtype of DMTFs, was overexpressed in breast cancer tissues and promotes tumorigenesis in a transgenic mouse model [45]. Niklaus et al. indicated the cisplatin resistance of breast cancer cells is associated with expression of DMTF1- $\beta$ by using SKBR3 (cisplatin sensitive) and MCF7 (cisplatin resistant) breast cancer cell lines in vitro [46]. Maglic et al. demonstrated that overexpression of $D M T F 1 \beta$ was associated with poor clinical outcomes, by examining the expression of DMTF $1 \beta$ in the cancer and adjacent tissue from twenty breast cancer patients, which suggested that DMTF1 $\beta$ could be considered a potential diagnostic index for patients with breast cancer [47]. When it comes to RORA, viewed as a member of the circadian genes, it could disrupt endogenous homeostasis and thereby promote endocrine tumor development and accelerate progression resulting from the dysfunction of this gene [48]. Taheri et al. found that one functional polymorphism (rs4774388) of RORA was associated with breast cancer risk after performing a comparative analysis between the breast cancer patients and the healthy persons in Iran [49]. Besides, $\mathrm{Du}$ and $\mathrm{Xu}$ [50] observed that RORA suppressed the expression of malignant phenotypes in breast cancer cell lines both in vitro and in vivo, which indicated that RORA could be considered an ideal potential diagnostic biomarker and therapeutic target of breast cancer. In the module 2, MATR3, known as a vital pathogenic gene of amyotrophic lateral sclerosis, is still poorly understood in the process of cancerization [51]. Just a few studies were conducted; for example, Yang et al. performed Western blot and RNA immunoprecipitation assay to find that the lncRNA SNHG1 was directly interacted with MATR3 to promote neuroblastoma progression [52]. Nho et al. observed the "Licochalcone H" could suppress cell viability and induce apoptosis in human oral squamous cell lines by suppression of MATR3 [53]. In short, previous articles indicated that the hub genes involved in the two modules showed a variety of physiological and pathological functions in breast cancer as an integrated interaction network including lncRNAs and TFs, which have significant prognosis capability and could be used as prognostic signatures of breast cancer.

Furthermore, TF motif searching analysis was performed to demonstrated that TFs might bind to the enhancers or promoters of important hub lncRNAs and form "feedback loops" to participate in cancer biology. The enriched pathways were shown to be closely associated with breast cancer; for example, the thyroid hormone signaling pathway ranked as having the highest degree of enrichment. Numerous studies were conducted to study the close relationship between the thyroid hormone and breast cancer. Hercbergs et al. indicated that thyroid hormone promoted the proliferation of the breast cells in vitro and breast cancer cases with hypothyroid function were less likely to be associated with lymph node metastases [54]. Søgaard et al. pointed out that hyperthyroidism was a risk factor for the incidence of breast cancer based on a population-based cohort study [55]. Besides, the NFkappa B signaling pathway ranked the top 10 signaling pathway in our analysis, indicating a vital role in the regulation of breast cancerization. Liu et al. showed that $\operatorname{lncRNA}$ NKILA could block the phosphorylation of $I \kappa B$ in vitro and suppress the metastasis of breast cancer by comparison of the different expressions of NKILA between the benign breast tissues and invasive carcinomas [56].

In summary, we provided a comprehensive analysis of breast cancer-related IncRNA-TF ceRNA crosstalk. The results demonstrated that the synergistic, competitive lncRNA-TF pairs played important roles in pathological processes of breast cancer and had strong effect on the prognosis of breast cancer patients. Although our study showed valuable results associated with breast cancer, there were still some limitations. First, we integrated hypergeometric test and PCC computed by gene expression profile to identify significant $\operatorname{lncRNA-TF}$ interactions. A stricter measure will decrease false-positive rate and increase accuracy and reliability of our results. Second, we only used FANTOM5 enhancer data to investigate the regulatory loops between TFs and lncRNA enhancers. If we can download the samesample multiomics data from TCGA, the core lncRNA-TF feedback loops would be more accurate. Third, in this study, we conducted a bioinformatics analysis to identify the crucial factors in breast cancer; results indicated that some genes (TFs or lncRNAs) might play vital roles in the subtype cancers. These results also encouraged us to validate the biological function and mechanism. In further study, we will conduct the biological experiments to investigate these potential factors. In a word, the identified lncRNAs and TFs in the global lncRNA-TF subnetwork and closely connected modules would provide important information for further breast cancer studies and be worth the experimental validations.

\section{Data Availability}

The raw data used to support the findings of this study are available from the Supplementary Materials.

\section{Conflicts of Interest}

The authors declare that they have no conflicts of interest to disclose.

\section{Authors' Contributions}

Jian Qiu is responsible for ensuring that the descriptions are accurate and agreed by all authors. Jian Qiu designed this 
study. Xinrong Li and Junquan Zhu collected and processed data. Xinrong Li wrote the manuscript.

\section{Supplementary Materials}

Supplementary 1. Supplementary Table S1: raw data for processed mRNA expression from TCGA.

Supplementary 2. Supplementary Table S2: raw data for processed lnc expression from TCGA.

Supplementary 3. Supplementary Table S3: raw data for miRNA-mRNA interactions from StarBase.

Supplementary 4. Supplementary Table S4: raw data for hypergeometric results between lncRNAs and TFs.

Supplementary 5. Supplementary Table S5: raw data for PCC results between lncRNAs and TFs.

Supplementary 6. Supplementary Table S6: raw data for network interactions in Figure 2(a).

Supplementary 7. Supplementary Table S7: raw data for clinical information of TCGA breast cancer.

Supplementary 8. Supplementary Table S8: topology feature of genes of the lncRNA-TF-associated ceRNA network.

\section{References}

[1] C. E. DeSantis, J. Ma, A. Goding Sauer, L. A. Newman, and A. Jemal, "Breast cancer statistics, 2017, racial disparity in mortality by state," CA: a Cancer Journal for Clinicians, vol. 67, no. 6, pp. 439-448, 2017.

[2] B. Weigelt, J. L. Peterse, and L. J. van't Veer, "Breast cancer metastasis: markers and models," Nature Reviews Cancer, vol. 5, no. 8, pp. 591-602, 2005.

[3] Y. Miki, J. Swensen, D. Shattuck-Eidens et al., "A strong candidate for the breast and ovarian cancer susceptibility gene BRCA1," Science, vol. 266, no. 5182, pp. 66-71, 1994.

[4] M. R. Stratton and N. Rahman, "The emerging landscape of breast cancer susceptibility," Nature Genetics, vol. 40, no. 1, pp. 17-22, 2008.

[5] S. Jathar, V. Kumar, J. Srivastava, and V. Tripathi, "Technological developments in lncRNA biology," Advances in Experimental Medicine and Biology, vol. 1008, pp. 283-323, 2017.

[6] R. A. Gupta, N. Shah, K. C. Wang et al., "Long non-coding RNA_HOTAIR_ reprograms chromatin state to promote cancer metastasis," Nature, vol. 464, no. 7291, pp. 10711076, 2010.

[7] G. Arun, S. Diermeier, M. Akerman et al., "Differentiation of mammary tumors and reduction in metastasis upon Malat1 lncRNA loss," Genes \& Development, vol. 30, no. 1, pp. 3451,2016

[8] H. Dong, W. Wang, S. Mo et al., "SP1-induced IncRNA AGAP2-AS1 expression promotes chemoresistance of breast cancer by epigenetic regulation of MyD88," Journal of Experimental \& Clinical Cancer Research, vol. 37, no. 1, p. 202, 2018.

[9] S. Ergun and S. Oztuzcu, "Oncocers: ceRNA-mediated crosstalk by sponging miRNAs in oncogenic pathways," Tumor Biology, vol. 36, no. 5, pp. 3129-3136, 2015.

[10] X. Qi, D. H. Zhang, N. Wu, J. H. Xiao, X. Wang, and W. Ma, "ceRNA in cancer: possible functions and clinical implica- tions," Journal of Medical Genetics, vol. 52, no. 10, pp. 710718, 2015.

[11] X. Wang, S. Zhang, and H. Qian, "Discovery of significant pathways in breast cancer metastasis via module extraction and comparison," IET Systems Biology, vol. 8, no. 2, pp. 47$55,2014$.

[12] A. L. Barabási, N. Gulbahce, and J. Loscalzo, "Network medicine: a network-based approach to human disease," Nature Reviews Genetics, vol. 12, no. 1, pp. 56-68, 2011.

[13] S. M. Hill, The HPN-DREAM Consortium, L. M. Heiser et al., "Inferring causal molecular networks: empirical assessment through a community-based effort," Nature Methods, vol. 13, no. 4 , pp. 310-318, 2016.

[14] C. J. Holtz-Heppelmann, A. Algeciras, A. D. Badley, and C. V. Paya, "Transcriptional regulation of the human FasL promoter-enhancer region," The Journal of Biological Chemistry, vol. 273, no. 8, pp. 4416-4423, 1998.

[15] D. Vella, S. Marini, F. Vitali, D. Di Silvestre, G. Mauri, and R. Bellazzi, "MTGO: PPI network analysis via topological and functional module identification," Scientific Reports, vol. 8, no. 1, p. 5499, 2018.

[16] A. Frankish, M. Diekhans, A. M. Ferreira et al., "GENCODE reference annotation for the human and mouse genomes," Nucleic Acids Research, vol. 47, no. D1, pp. D766-d773, 2019.

[17] G. D. Bader and C. W. Hogue, “An automated method for finding molecular complexes in large protein interaction networks," BMC Bioinformatics, vol. 4, no. 1, p. 2, 2003.

[18] R. Andersson, The FANTOM Consortium, C. Gebhard et al., "An atlas of active enhancers across human cell types and tissues," Nature, vol. 507, no. 7493, pp. 455-461, 2014.

[19] The FANTOM Consortium and the RIKEN PMI and CLST (DGT), "A promoter-level mammalian expression atlas," Nature, vol. 507, no. 7493, pp. 462-470, 2014.

[20] C. E. Grant, T. L. Bailey, and W. S. Noble, "FIMO: scanning for occurrences of a given motif," Bioinformatics, vol. 27, no. 7, pp. 1017-1018, 2011.

[21] M. Wade, Y.-C. Li, and G. M. Wahl, "MDM2, MDMX and p53 in oncogenesis and cancer therapy," Nature Reviews Cancer, vol. 13, no. 2, pp. 83-96, 2013.

[22] S. Haupt, D. Buckley, J. M. Pang et al., "Targeting Mdmx to treat breast cancers with wild-type p53," Cell Death \& Disease, vol. 6, no. 7, article e1821, 2015.

[23] C. Gao, G. Xiao, A. Piersigilli, J. Gou, O. Ogunwobi, and J. Bargonetti, "Context-dependent roles of MDMX (MDM4) and MDM2 in breast cancer proliferation and circulating tumor cells," Breast Cancer Research, vol. 21, no. 1, p. 5, 2019.

[24] J. A. Benanti, D. K. Williams, K. L. Robinson, H. L. Ozer, and D. A. Galloway, "Induction of extracellular matrix-remodeling genes by the senescence-associated protein APA-1," Molecular and Cellular Biology, vol. 22, no. 21, pp. 7385-7397, 2002.

[25] Z. Du, T. Sun, E. Hacisuleyman et al., "Integrative analyses reveal a long noncoding RNA-mediated sponge regulatory network in prostate cancer," Nature Communications, vol. 7, no. 1, article 10982, 2016.

[26] M. Li, X. Wu, J. Wang, and Y. Pan, "Towards the identification of protein complexes and functional modules by integrating PPI network and gene expression data," BMC Bioinformatics, vol. 13, no. 1, p. 109, 2012.

[27] S. Haupt, J. O. Mejía-Hernández, R. Vijayakumaran, S. P. Keam, and Y. Haupt, "The long and the short of it: the 
MDM4 tail so far," Journal of Molecular Cell Biology, vol. 11, no. 3, pp. 231-244, 2019.

[28] E. A. Fry and K. Inoue, "c-MYB and DMTF1 in cancer," Cancer Investigation, vol. 37, no. 1, pp. 46-65, 2019.

[29] M. J. Wortman, L. K. Hanson, L. Martínez-Sobrido et al., "Regulation of PURA gene transcription by three promoters generating distinctly spliced 5-prime leaders: a novel means of fine control over tissue specificity and viral signals," BMC Molecular Biology, vol. 11, no. 1, p. 81, 2010.

[30] M. J. Zeitz, K. S. Malyavantham, B. Seifert, and R. Berezney, "Matrin 3: chromosomal distribution and protein interactions," Journal of Cellular Biochemistry, vol. 108, no. 1, pp. 125-133, 2009.

[31] Y. Qu, N. Gharbi, X. Yuan et al., “Axitinib blocks Wnt/ $\beta$ catenin signaling and directs asymmetric cell division in cancer," Proceedings of the National Academy of Sciences of the United States of America, vol. 113, no. 33, pp. 9339-9344, 2016.

[32] C. Küper, F. X. Beck, and W. Neuhofer, "NFAT5-mediated expression of S100A4 contributes to proliferation and migration of renal carcinoma cells," Frontiers in Physiology, vol. 5, p. 293, 2014.

[33] G. Gu, L. Gelsomino, K. R. Covington et al., "Targeting thyroid hormone receptor beta in triple-negative breast cancer," Breast Cancer Research \& Treatment, vol. 150, no. 3, pp. 535-545, 2015.

[34] A. Kaymak, S. Sayols, T. Papadopoulu, and H. Richly, "Role for the transcriptional activator ZRF1 in early metastatic events in breast cancer progression and endocrine resistance," Oncotarget, vol. 9, no. 47, pp. 28666-28690, 2018.

[35] K. L. Thu, I. Soria-Bretones, T. W. Mak, and D. W. Cescon, "Targeting the cell cycle in breast cancer: towards the next phase," Cell Cycle, vol. 17, no. 15, pp. 1871-1885, 2018.

[36] Z. Liu, L. Jiang, G. Liang et al., "Hepatitis B virus reactivation in breast cancer patients undergoing chemotherapy: a review and meta-analysis of prophylaxis management," Journal of Viral Hepatitis, vol. 24, no. 7, pp. 561-572, 2017.

[37] Y. C. Liu, C. T. Yeh, and K. H. Lin, "Molecular functions of thyroid hormone signaling in regulation of cancer progression and anti-apoptosis," International Journal of Molecular Sciences, vol. 20, no. 20, article 4986, 2019.

[38] M. Mirabelli, E. Chiefari, B. Arcidiacono et al., "Mediterranean diet nutrients to turn the tide against insulin resistance and related diseases," Nutrients, vol. 12, no. 4, p. 1066, 2020.

[39] M. Samson, N. Porter, O. Orekoya et al., "Progestin and breast cancer risk: a systematic review," Breast Cancer Research and Treatment, vol. 155, no. 1, pp. 3-12, 2016.

[40] R. Yang, L. Xing, M. Wang, H. Chi, L. Zhang, and J. Chen, "Comprehensive analysis of differentially expressed profiles of lncRNAs/mRNAs and miRNAs with associated ceRNA networks in triple-negative breast cancer," Cellular Physiology and Biochemistry, vol. 50, no. 2, pp. 473-488, 2018.

[41] S. Haupt, R. Vijayakumaran, P. J. Miranda, A. Burgess, E. Lim, and Y. Haupt, "The role of MDM2 and MDM4 in breast cancer development and prevention," Journal of Molecular Cell Biology, vol. 9, no. 1, pp. 53-61, 2017.

[42] V. A. de Weger, M. de Jonge, M. H. G. Langenberg et al., "A phase I study of the HDM2 antagonist SAR405838 combined with the MEK inhibitor pimasertib in patients with advanced solid tumours," British Journal of Cancer, vol. 120, no. 3, pp. 286-293, 2019.
[43] M. M. Gounder, T. M. Bauer, G. K. Schwartz et al., "A phase 1 study of the MDM2 inhibitor DS-3032b in patients (pts) with advanced solid tumors and lymphomas," Journal of Clinical Oncology, vol. 34, article 2581, Supplement 15, 2016.

[44] F. Meric-Bernstam, M. N. Saleh, J. R. Infante et al., "Phase I trial of a novel stapled peptide ALRN-6924 disrupting MDMX- and MDM2-mediated inhibition ofWT p53in patients with solid tumors and lymphomas," Journal of Clinical Oncology, vol. 35, 15_suppl, p. 2505, 2017.

[45] N. Tian, J. Li, J. Shi, and G. Sui, "From general aberrant alternative splicing in cancers and its therapeutic application to the discovery of an oncogenic DMTF1 isoform," International Journal of Molecular Sciences, vol. 18, no. 3, p. 191, 2017.

[46] N. J. Niklaus, M. Humbert, and M. P. Tschan, "Cisplatin sensitivity in breast cancer cells is associated with particular DMTF1 splice variant expression," Biochemical and Biophysical Research Communications, vol. 503, no. 4, pp. 2800-2806, 2018.

[47] D. Maglic, D. B. Stovall, J. M. Cline et al., "DMP1 $\beta$, a splice isoform of the tumour suppressor DMP1 locus, induces proliferation and progression of breast cancer," The Journal of Pathology, vol. 236, no. 1, pp. 90-102, 2015.

[48] S. Morales-Santana, S. Morell, J. Leon, A. Carazo-Gallego, J. C. Jimenez-Lopez, and M. Morell, "An overview of the polymorphisms of circadian genes associated with endocrine cancer," Frontiers in Endocrinology, vol. 10, p. 104, 2019.

[49] M. Taheri, M. D. Omrani, R. Noroozi, S. Ghafouri-Fard, and A. Sayad, "Retinoic acid-related orphan receptor alpha (RORA) variants and risk of breast cancer," Breast Disease, vol. 37, no. 1, pp. 21-25, 2017.

[50] J. Du and R. Xu, "ROR $\alpha$, a potential tumor suppressor and therapeutic target of breast cancer," International Journal of Molecular Sciences, vol. 13, no. 12, pp. 15755-15766, 2012.

[51] C. S. Leblond, Z. Gan-Or, D. Spiegelman et al., "Replication study of MATR3 in familial and sporadic amyotrophic lateral sclerosis," Neurobiol Aging, vol. 37, pp. 209.e217-209.e221, 2016.

[52] T. W. Yang, D. Sahu, Y. W. Chang, C. L. Hsu, and C. H. Hsieh, "RNA-binding proteomics reveals MATR3 interacting with lncRNA SNHG1 to enhance neuroblastoma progression," Journal of Proteome Research, vol. 18, no. 1, pp. 406-416, 2018.

[53] S. H. Nho, G. Yoon, J. H. Seo et al., "Licochalcone H induces the apoptosis of human oral squamous cell carcinoma cells via regulation of matrin 3," Oncology Reports, vol. 41, no. 1, pp. 333-340, 2019.

[54] A. Hercbergs, S. A. Mousa, M. Leinung, H. Y. Lin, and P. J. Davis, "Thyroid hormone in the clinic and breast cancer," Hormones and Cancer, vol. 9, no. 3, pp. 139-143, 2018.

[55] M. Søgaard, D. K. Farkas, V. Ehrenstein, J. O. Jørgensen, O. M. Dekkers, and H. T. Sørensen, "Hypothyroidism and hyperthyroidism and breast cancer risk: a nationwide cohort study," European Journal of Endocrinology, vol. 174, no. 4, pp. 409414, 2016.

[56] B. Liu, L. Sun, Q. Liu et al., “A cytoplasmic NF- $\kappa$ B interacting long noncoding RNA blocks $\mathrm{I} \kappa \mathrm{B}$ phosphorylation and suppresses breast cancer metastasis," Cancer Cell, vol. 27, no. 3, pp. 370-381, 2015. 